\title{
Noninvasive quantitative estimation of hepatic steatosis by ultrasound: a comparison of the hepato-renal index and ultrasound attenuation index
}

\author{
Heon-Ju Kwon', Kyoung Won Kim², Jin-Hee Jung ${ }^{3}$, Sang Hyun Choi ${ }^{2}$, Woo Kyoung Jeong ${ }^{4}$, \\ Bohyun Kim ${ }^{5}$, Gi-Won Song ${ }^{6}$, Sung-Gyu Lee ${ }^{6}$
}

${ }^{1}$ Department of Radiology, Kangbuk Samsung Hospital, Sungkyunkwan University School of Medicine, Seoul, ${ }^{2}$ Department of Radiology, Asan Medical Center, University of Ulsan College of Medicine, Seoul, ${ }^{3}$ Department of Radiology, Dongguk University Ilsan Medical Center, Gyeonggi-do, ${ }^{4}$ Department of Radiology and Center for Imaging Science, Samsung Medical Center, Sungkyunkwan University School of Medicine, Seoul, ${ }^{5}$ Department of Radiology, Ajou University Medical Center, Gyeonggi-do, ${ }^{6}$ Department of Liver Transplantation Surgery, Asan Medical Center, University of Ulsan College of Medicine, Seoul, Korea

\begin{abstract}
Aims: To compare the accuracy of the ultrasound attenuation index (USAI) and hepato-renal index (HRI) for the diagnosis of hepatic steatosis (HS). Material and methods: Two hundred and twenty-four potential living hepatic donors underwent US and subsequent US-guided liver biopsy. The USAI was calculated from US images with an $8 \mathrm{MHz}$ transducer and HRI was measured on sagittal images with a clear visualization of both the liver and kidney. Using histological degrees of HS as the reference standard, we compared the performance of USAI and HRI for diagnosing HS $\geq 5 \%$ and $\geq 30 \%$ by receiver operating characteristic curve analysis. The interobserver agreement was evaluated by using intraclass correlation coefficients (ICCs) or Bland-Altman statistics. Results: Histologic degree of HS was 0-70\% (median, 5\%). HRI showed a tendency towards higher accuracy than USAI for diagnosing HS $\geq 5 \%$ (the area under the ROC curve, 0.856 vs. $0.820 ; p=0.279$ ) and $\geq 30 \%$ ( 0.937 vs. $0.909 ; \mathrm{p}=0.378)$ without statistical significance. There was an excellent interobserver agreement for both USAI and HRI ( $\mathrm{ICC}=0.931$ and 0.973 , respectively). According to the Bland-Altman method, the $95 \%$ limits of difference between two readers for HS were $-8.5 \%$ to $6.6 \%$ by USAI and $-4.8 \%$ to $6.2 \%$ by HRI. Most patients would have the difference of calculated HS by USAI (74.0\%) and HRI (96.0\%) from different operators within a range of $\pm 5 \%$. Conclusions: Although statistically insignificant, HRI was superior to USAI for the diagnosis and quantitative estimation of HS in terms of diagnostic performance, including accuracy and reproducibility.
\end{abstract}

Keywords: non-alcoholic fatty liver disease, hepato-renal index, ultrasound attenuation index, ultrasonography, fatty liver

\section{Introduction}

Non-alcoholic fatty liver disease (NAFLD) is rapidly becoming the most common liver disease worldwide,

Received 02.04.2016, Accepted 15.06.2016

Med Ultrason

2016, Vol. 18, No 4, 431-437

Corresponding author: Kyoung Won Kim MD,

Department of Radiology,

Asan Medical Center, University of Ulsan

College of Medicine,

86 Asanbyeongwon-gil, Songpa-gu,

Seoul 05505, South Korea

Phone: +82-2-30104385, Fax: +82-2-4764719

E-mail:kimkw@amc.seoul.kr raising serious clinical and public health concerns [1]. In recent years, it has emerged as an important independent risk factor for insulin resistance, cardiovascular disease, and metabolic syndrome $[2,3]$. In addition, it may progress to steatohepatitis, fibrosis, and even life-threatening liver disease, such as cirrhosis and hepatocellular carcinoma $[4,5]$. Therefore, to prevent NAFLD progression, screening and surveillance strategies are essential prerequisites. Because of the occult nature of this disease, a method for accurate assessment of hepatic fat infiltration would have profound significance in the clinical setting.

Liver biopsy is the "gold" standard for quantification of hepatic steatosis. However, due to its high prevalence 
and the asymptomatic features of NAFLD in the general population, using routine liver biopsy to diagnose NAFLD is unreasonable. In addition, since biopsy is an invasive procedure with potential risks of complications that range from minor pain at the biopsy site to more serious events such as hemorrhage and death [6], there has been a great deal of effort invested in developing accurate, simple, cost-effective, and noninvasive diagnostic imaging methods that can quantitatively measure hepatic steatosis.

In particular, ultrasound (US) has been widely used to detect fatty infiltration of the liver in that it is non-radioactive, inexpensive, and readily available. Usually, US for the diagnosis of NAFLD is based mainly on a subjective assessment of liver echogenicity [7-9]. However, this method is operator-dependent with high inter- and intraobserver variability [10-12]. Thus, recently, several attempts have been made to establish objective methods for quantitative assessment of hepatic steatosis by US. To date, few studies have assessed the utility of the US attenuation index (USAI) or the hepato-renal index (HRI) as tools for quantification of hepatic steatosis [7,13-15]. In the available studies, these quantitative parameters show good performance for the diagnosis of NAFLD and good correlation with hepatic steatosis. However, until now, there has been no study that has directly compared their accuracy for the diagnosis of NAFLD and quantifying hepatic steatosis in a large human population using hepatic biopsy as the reference standard.

Given the growing understanding and interest in the importance of NAFLD, it may be valuable to assess these indices' relative accuracy to determine the proper quantitative measurement of hepatic steatosis that would be useful for screening, follow-up, and evaluation of treatment response. The purpose of our study was therefore to compare the accuracy of USAI and HRI for the diagnosis and quantitative estimation of hepatic steatosis using liver biopsy as the reference standard.

\section{Material and methods}

Institutional review board approval was obtained for this study and informed patient consent was waived owing to the retrospective nature of the study.

\section{Study population}

Of the potential living hepatic donors between January and August 2014, those who met the following criteria underwent US and subsequent US-guided needle biopsy for the evaluation of hepatic steatosis and the presence of other occult liver disease: the absence of any documented liver disease, negative serologic findings for hepatitis B or $\mathrm{C}$, and aspartate aminotransferase or alanine aminotrans- ferase levels less than three times the upper normal limit. A radiologist (blinded) reviewed their clinical information and US images for exclusion. Of the 248 consecutive potential donors, 24 donor candidates were excluded from our study due to absence of images including both kidney and liver $(\mathrm{n}=10)$, heterogeneous distribution of fatty infiltration in the liver $(\mathrm{n}=1)$, proteinuria or increased creatinine level $(\mathrm{n}=3)$, or subjects' heavy alcohol consumption (over $210 \mathrm{~g} /$ week in men and $140 \mathrm{~g} /$ week in women) $(\mathrm{n}=10)$. The remaining 224 study patients consisted of 146 men (mean age \pm standard deviation [SD], 28.9 \pm 8.6 years old; range, 18-56) and 78 women (34.3 \pm 10.4 years old; 18-55). For all study patients, anthropometric data, liver function test results (obtained 0-40 days [median, 3 days] prior to the imaging examination), and the amount of weekly alcohol intake were recorded. Height and body weight were recorded to the nearest $0.1 \mathrm{~cm}$ and the nearest $0.1 \mathrm{~kg}$, respectively, in all study subjects. The body mass index was calculated as body weight in kilograms divided by height in meters squared $\left(\mathrm{kg} / \mathrm{m}^{2}\right)$.

\section{US examination}

Prior to the biopsy, the target site was scanned by one of five board-certified abdominal radiologists using a Sequoia 512 US machine (Acuson, Mountain View, CA, USA) equipped with an 8-12 $\mathrm{MHz}$ linear array transducer and a 1-4 MHz convex array transducer. In general, patients received nothing by mouth for at least 8 hours before the examination. The examinations were performed in the supine position while the subjects were holding their breath. For HRI measurement, images were obtained with a frequency of $4 \mathrm{MHz}$; all of the instrument settings, including "gain", "depth", and "time-gain compensation" were optimized for each image. For USAI measurements, images were obtained with a frequency of $8 \mathrm{MHz}$; the time-gain compensation was inactivated, brightness was adjusted only by the gain, and the focal zone was adjusted to be in the deepest position.

\section{US image analysis}

The same radiologist who reviewed the subjects for inclusion selected two sets of US images for each subject. The first set was US images obtained using a linear transducer for the calculation of USAI, and the second set was a sagittal image clearly visualizing both liver and kidney and was obtained using a convex transducer for the calculation of HRI. Then, the image sets were randomly assorted after removing the subjects' information from the images. Another 8 year experienced abdominal radiologist (blinded), who was blinded to the radiology report and histopathology diagnosis calculated USAI and HRI. All images were reviewed on a Picture Archiving Communication System workstation monitor (blinded). To assess agreement in these quantitative measurements 
between different readers, 50 patients were randomly selected and reviewed by another radiologist (blinded, 5 years' experience) who did not know the results of the initial measurement and biopsy.

To measure the USAI (fig 1a), the observer drew two $3 \times 3 \mathrm{~mm}$ square regions of interest (ROI) in superficial (3-cm depth) and deep (5-cm depth) areas from the body surface on the sonogram, avoiding vessels, noise, and artifacts. In the liver, the ROI corresponded to the site where the biopsy was performed. To avoid confounding factors that could modify the echo intensity and thus bias comparisons, the mean echogenicities of both the superficial and deep areas were obtained on the same longitudinal US plane. The mean echogenicity within the ROI was measured on a 256-level gray scale $(0-255)$. The USAI was then defined as the difference between the superficial and deep echogenicity/superficial echogenicity $\times 100$. For the assessment of HRI (fig 1b), a circular ROI of $100 \mathrm{~mm}^{2}$ in the liver parenchyma was selected. The ROI was selected to contain an area of as uniform echogenicity as possible, excluding large blood vessels or bile ducts. Another ROI of $33 \mathrm{~mm}^{2}$ was identified in the right renal cortex with no large vessels, renal sinus, or medulla. To avoid the interference of depth-dependent echo-intensity attenuation, the liver and right kidney ROIs were selected at the same depth on the US images. The gray scale mean value of the pixels within the two ROIs was used as a measurement of echo intensity. Then we divided the average hepatic gray scale by the average renal cortex gray scale to calculate HRI.

\section{Reference standard}

The same five radiologists who performed the US examination performed US-guided liver biopsies using a freehand technique; each of the five radiologists has experience performing at least 100 US-guided liver biopsies. Each biopsy was performed using an 18-gauge needle (Stericut 18G coaxial, TSK Laboratory, Tochigi, Japan). In all subjects, biopsy specimens were obtained twice at two different sites located approximately among hepatic segments V, VI, VII, and VIII. Biopsy sites were devoid of macroscopic vessels and were approximately 1 $\mathrm{cm}$ apart. Each biopsy specimen was approximately 1.5 $\mathrm{cm}$ in length. An experienced liver pathologist who had no knowledge of the radiologic findings reviewed the histologic findings. The slides for histologic review were prepared with hematoxylin-eosin and Masson trichrome staining. The degree of steatosis was quantified by using a scale that was based on percentages (i.e., the amount of liver parenchyma replaced by steatotic droplets) [16]. For our study, the average of the degree of total hepatic steatosis in two biopsy specimens was used as the reference standard.

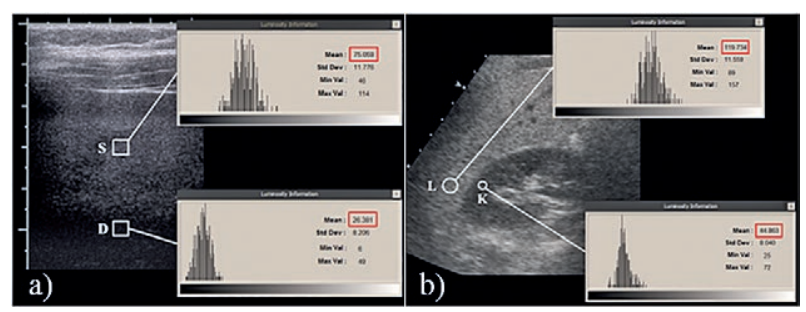

Fig 1. Ultrasound (US) images of the liver: a) Measurement of US attenuation index. On the sonogram, an observer drew two $3 \times 3 \mathrm{~mm}$ square regions of interest (ROI, squares) in the superficial (S; 3-cm depth) and deep (D; 5-cm depth) areas, avoiding vessels, noise, and artifacts. The mean echogenicities within the ROIs were measured on a 256-level gray scale (0-255). The US attenuation index is 64.853: (75.059$26.381) / 75.059 \times 100=64.853$; b) Measurement of hepato-renal index. On the sonogram, an observer drew a circular ROI of $100 \mathrm{~mm}^{2}$ in the liver parenchyma (L) and another ROI of $33 \mathrm{~mm}^{2}$ in the right renal cortex $(\mathrm{K})$ at the same depth. The hepato-renal index is 2.669: $119.734 / 44.863=2.669$.

\section{Statistical analysis}

All statistical analyses were performed using SPSS software version 18.0 for Windows (SPSS, Chicago, IL, USA) and MedCalc version 11.2.1 software for Windows (Medcalc Software, Mariakeke, Belgium). The accuracy of USAI and HRI for quantitative estimation of hepatic steatosis was analyzed by comparing the estimated hepatic steatosis by linear regression models and the degree of hepatic steatosis at histologic analysis. Bland-Altman 95\% limits of agreement between the estimated and the histologic degree of hepatic steatosis were obtained. The diagnostic accuracy of USAI and HRI in the diagnosis of hepatic steatosis $\geq 5 \%$ and $\geq 30 \%$ [17-19] was evaluated and compared using receiver operating characteristic (ROC) curve analysis. The optimal cutoff values of USAI and HRI for diagnosing hepatic steatosis $\geq 5 \%$ and $\geq 30 \%$ were obtained from the Youden index (maximum [sensitivity + specificity - 1]) [20], and corresponding sensitivity, specificity, positive and negative predictive values (PPV and NPV) were calculated for the chosen cutoff values. Then, the sensitivities and specificities were compared between USAI and HRI using the McNemar test. The interobserver agreement was evaluated by using intraclass correlation coefficients (ICCs) or Bland-Altman statistics [21,22]. An ICC of less than 0.40 signified poor agreement; those of $0.40-0.75$, fair to good (moderate) agreement; and those of 0.76-1.00, excellent agreement [23]. Values for $\mathrm{p}<0.05$ were considered to indicate a significant difference.

\section{Results}

The characteristics of the study population are summarized in Table I. The histologic degree of hepatic 
steatosis was $0-70 \%$ (median, 5\%); 91 (40.6\%) patients had mild hepatic steatosis $(\geq 5 \%$ and $<30 \%)$ and 24 $(10.7 \%)$ patients had moderate or severe hepatic steatosis $(\geq 30 \%)$, respectively. Among them, only one patient had histologic findings of non-alcoholic steatohepatitis. The USAI ranged from -24.129 to 73.037 (mean \pm SD, $22.507 \pm 15.876)$ and HRI ranged from 0.701 to 3.720 (1.445 \pm 0.473$)$, respectively.

The scatter plots between the histologic degree of hepatic steatosis and these measurements are presented in fig 2 . Both USAI and HRI were highly positively associated with the histologic degree of fatty liver, with $\gamma=0.682$ $(p<0.001)$ and $\gamma=0.853(p<0.001)$, respectively. A stronger linear relationship was found between the HRI and the histologic degree of hepatic steatosis compared with that between the USAI and the histologic degree of hepatic steatosis. The prediction models of hepatic steatosis using USAI and HRI by linear regression analysis are presented in table II. The Bland-Altman 95\% limits of agreement

Table I. Clinical and histological characteristics of study population.

\begin{tabular}{ll}
\hline Characteristics & $\begin{array}{l}\text { Study population } \\
(\mathbf{n = 2 2 4})\end{array}$ \\
\hline Age (years) & \\
$\quad$ Total & $30.8 \pm 9.6(18-56)$ \\
Men & $28.9 \pm 8.6(18-56)$ \\
$\quad$ Women & $34.3 \pm 10.4(18-55)$ \\
Sex & $146(65.2 \%)$ \\
$\quad$ Men & $78(34.8 \%)$ \\
Women & $23.1 \pm 3.1(16.1-35.7)$ \\
Body mass index (kg/m2) & $20.9 \pm 8.6(11-111)$ \\
AST (normal range: $0-40$ UI/L) & $19.9 \pm 11.9(1-88)$ \\
ALT (normal range: $0-40$ UI/L) & \\
Alcohol intake & $97(43.3 \%)$ \\
$\quad$ None & $127(56.7 \%)$ \\
Social drinker* & \\
Hepatic steatosis at histological analysis & \\
$\quad \geq 5 \%$ & $115(51.3 \%)$ \\
$\geq 30 \%$ & $24(10.7 \%)$ \\
\hline
\end{tabular}

The values are presented as mean \pm standard deviation (range) or number (percentage); *Social drinker is defined as the amount of alcohol consumption is $\leq 210 \mathrm{~g} /$ week in men and $\leq 140 \mathrm{~g} /$ week in women; AST- aspartate aminotransferase, ALT- alanine aminotransferase. between the estimated and the histologic degree of hepatic steatosis were $-19.8 \%$ to $19.8 \%$ for USAI (the estimated hepatic steatosis $=0.594 \times$ USAI -3.559$)$ and $-14.1 \%$ to $14.1 \%$ for HRI (the estimated hepatic steatosis $=24.916 \times \mathrm{HRI}-26.212$ ), indicating the higher accuracy of HRI in quantitatively estimating hepatic steatosis.

For the diagnosis of hepatic steatosis $\geq 5 \%$, the areas under the ROC curve of USAI and HRI were 0.820 (95\% confidence interval [CI], 0.764-0.868) and $0.856(95 \%$ CI, 0.803-0.899), respectively (fig 3a). For the diagnosis of hepatic steatosis $\geq 30 \%$, the areas under the ROC curve of USAI and HRI were 0.909 (95\% CI, 0.863-0.943) and 0.937 (95\% CI, 0.897-0.965), respectively (fig 3b). Although the HRI showed a tendency towards a higher accuracy compared with USAI at both threshold levels of hepatic steatosis $\geq 5 \%$ and $\geq 30 \%$, the difference was not statistically significant $(\mathrm{p}=0.279$ for the diagnosis of hepatic steatosis $\geq 5 \%$ and $\mathrm{p}=0.378$ for the diagnosis of hepatic steatosis $\geq 30 \%$, respectively).

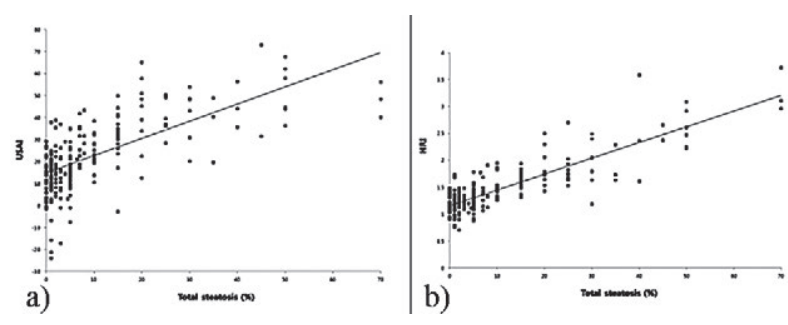

Fig 2. Correlation between a) ultrasound attenuation index and b) hepato-renal index, and the histologic degree of hepatic steatosis at liver biopsy.
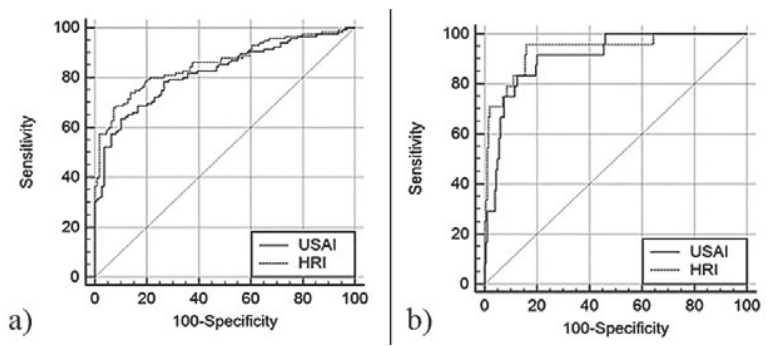

Fig 3. Receiver operating characteristic curves and area under the receiver operating characteristic curves of the hepato-renal index and ultrasound attenuation index are shown for the diagnosis of hepatic steatosis a) $\geq 5 \%$ and $b$ ) $\geq 30 \%$.

Table II. Linear regression models for hepatic steatosis estimation using USAI and HRI.

\begin{tabular}{llllll}
\hline & $\boldsymbol{\beta} \pm$ SD & Constant & p value & adj. $\mathbf{R}^{\mathbf{2}}$ & RMSE \\
\hline USAI & $0.594 \pm 0.043$ & $-3.559 \pm 1.175$ & $<0.001$ & $46.3 \%$ & 10.122 \\
HRI & $24.916 \pm 1.023$ & $-26.212 \pm 1.555$ & $<0.001$ & $72.7 \%$ & 7.223 \\
\hline
\end{tabular}

The values are presented as mean \pm standard deviation; USAI - ultrasound attenuation index, HRI- hepato-renal index, RMSE- root mean squared error. 
The sensitivity, specificity, PPV, and NPV of USAI and HRI with optimal cutoff values for diagnosing hepatic steatosis $\geq 5 \%$ and $\geq 30 \%$ are summarized in table III. For diagnosing hepatic steatosis $\geq 5 \%$, although both USAI and HRI had fairly good specificities $(89.9 \%$ and $92.7 \%$ ) and PPVs ( $86.9 \%$ and $90.7 \%$ ), they had relatively poor sensitivities $(63.5 \%$ and $67.8 \%)$ and NPVs $(70.0 \%$ and $73.2 \%$ ), respectively. The values of HRI were slightly higher than those of USAI, although the difference was statistically insignificant ( $\mathrm{p} \geq 0.486$ for all comparisons). For diagnosing hepatic steatosis $\geq 30 \%$, both USAI and HRI had excellent sensitivities $(91.7 \%$ and $95.8 \%)$ and NPVs (98.8\% and $99.4 \%)$, but they had relatively poor specificities $(80.0 \%$ and $83.5 \%)$ and poor PPVs $(35.5 \%$ and $41.1 \%$ ), respectively. The values of HRI were slightly higher than those of USAI but the difference was without statistical significance ( $\mathrm{p} \geq 0.281$ for all comparisons).

The 50 patients (28.6 \pm 9.4 years) who were randomly chosen for the analysis of interobserver agreement consisted of 34 men (24.9 \pm 5.9 years) and 16 women $(36.4 \pm 10.8$ years); their histological degree of hepatic steatosis ranged from 0 to $50 \%$ (mean, $8.6 \%$; median, $4.0 \%$ ). There were "excellent" interobserver agreements for USAI and for HRI (ICC $=0.931$ and 0.973 , respectively). According to the Bland-Altman method, the $95 \%$ limits of difference between two readers were $-8.5 \%$ to $6.6 \%$ for the estimated hepatic steatosis by USAI and $-4.8 \%$ to $6.2 \%$ for the estimated hepatic steatosis by HRI. If we tolerate a difference smaller than $5 \%$ for hepatic steatosis estimation, 37 $(74.0 \%)$ and $48(96.0 \%)$ patients would have a difference of calculated hepatic steatosis by USAI and HRI from different operators within the range of $\pm 5 \%$ hepatic steatosis.

\section{Discussions}

The routine sonographic interpretation of hepatic steatosis is generally based on a subjective impression of the hyperechogenicity of the liver parenchyma, fall of echogenicity with depth in the liver parenchyma, and poor or nonvisualization of the intrahepatic vessel borders and diaphragm. Thus, this interpretation is qualitative and prone to interobserver and intraobserver variance, which can lead to limited reproducibility and comparability $[10,11]$. Therefore, the development of an objective quantitative US measurement is important to obtain reproducibility and comparability.

In this study, we found that both USAI and HRI had good interobserver agreement, and the difference of the estimated hepatic steatosis between different operators mostly fell in the range of $\pm 5 \%$ hepatic steatosis. In previous studies, which investigated the correlation between USAI or HRI and histologic analysis $[7,13,14,24]$, they also showed that HRI or USAI was positively correlated with hepatic steatosis $(\gamma=0.71-0.884, p<0.001)$ and presented a large area under the ROC (0.87-1.00) for the diagnosis of hepatic steatosis, similar to the results of our study. And, their cutoff point of HRI for the diagnosis of hepatic steatosis $\geq 5 \%$ was also similar to that of our study. This similarity of results indicates reproducibility of these parameters across patients with various liver diseases, including NAFLD.

In this study, USAI and HRI were highly positively associated with the histologic degree of hepatic steatosis and very specific for the diagnosis of hepatic steatosis $\geq 5 \%$. In clinical settings of diagnosis for a mild degree of hepatic steatosis, a specific diagnosis is possible if USAI or HRI is greater than the cutoff value observed in the current study. However, its relatively low sensitivity may be an improper characteristic as a screening or surveillance method for NAFLD. Therefore, when USAI or HRI is within the normal limit, if mild hepatic steatosis is strongly suspected, a biopsy should be considered to eliminate false negative results of US. In contrast, USAI and HRI showed high sensitivity and low PPV for the

Table III. Diagnostic performance of USAI and HRI for diagnosing hepatic steatosis $\geq 5 \%$ and $\geq 30 \%$.

\begin{tabular}{cllll}
\hline & \multicolumn{2}{l}{ Hepatic steatosis $\geq \mathbf{5 \%}$} & Hepatic steatosis $\geq \mathbf{3 0 \%}$ \\
& USAI & HRI & USAI & HRI \\
\hline Cutoff value & 24.769 & 1.411 & 30.360 & 1.592 \\
Sensitivity & $63.5(73 / 115)$ & $67.8(78 / 115)$ & $91.7(22 / 24)$ & $95.8(23 / 24)$ \\
$95 \%$ CI & $54.7,72.3$ & $59.3,76.4$ & $80.6,102.7$ & $87.8,100.4$ \\
Specificity & $89.9(98 / 109)$ & $92.7(101 / 109)$ & $80.0(160 / 200)$ & $83.5(167 / 200)$ \\
$95 \%$ CI & $84.3,95.6$ & $87.8,97.6$ & $74.5-85.5$ & $78.4,88.6$ \\
PPV & $86.9(73 / 84)$ & $90.7(78 / 86)$ & $35.5(22 / 62)$ & $41.1(23 / 56)$ \\
$95 \%$ CI & $79.7,94.1$ & $84.6,96.8$ & $23.6,47.4$ & $28.2,54.0$ \\
NPV & $70.0(98 / 140)$ & $73.2(101 / 138)$ & $98.8(160 / 162)$ & $99.4(167 / 168)$ \\
$95 \%$ CI & $62.4,77.6$ & $65.8,80.6$ & $97.1,100.5$ & $98.2,100.6$ \\
\hline
\end{tabular}

The values are presented as percentages; Values in parentheses are the number of subjects used to calculate the percentage; USAI - ultrasound attenuation index, HRI - hepato-renal index, PPV - positive predictive value, NPV - negative predictive value. 
diagnosis of hepatic steatosis $\geq 30 \%$. Thus, in clinical settings of diagnosis for moderate to severe steatosis, these parameters can be effectively used as screening or surveillance diagnostic tools. However, because PPV is very low, false positive diagnoses occur very frequently. Therefore, in such a setting, it is better to use other highly specific non-invasive studies such as computed tomography or magnetic resonance imaging as ancillary methods. Therefore, it should be noted that the practical use of US parameters would be very restrictive in these settings.

In our study, we found that HRI showed higher accuracy in the quantitative estimation of hepatic steatosis with a stronger correlation with the histologic degree of hepatic steatosis than USAI. In addition, the diagnostic performance of HRI for hepatic steatosis $\geq 5 \%$ and $\geq 30 \%$ was also better than that of USAI although the difference was statistically insignificant. From a practical point of view, additional effort is required to obtain USAI, such as changing the US transducer from a low to a high frequency, which is not routinely used in abdominal US examination, as well as inactivating the time-gain compensation. Moreover, a retrospective analysis of USAI is impossible unless the images were obtained by a standardized protocol. On the contrary, HRI can be measured not only prospectively but also retrospectively from ordinary US images covering the liver and right kidney, obtained with routine scanning protocols and transducers.

Therefore, HRI might be clinically useful as an easy and relatively accurate method to diagnose hepatic steatosis. While there may be some limitations in the clinical use of HRI in that HRI cannot be measured in patients with disease in the right kidney or the right hepatic lobe, such pitfalls are not expected to occur frequently in the suspicious NAFLD patient group and can be easily recognized and avoided during the US image analysis.

There were a few limitations to our study. Firstly, the major limitation is the retrospective design and the calculation of the USAI and HRI indices from previously recorded US images. It is clear that under these conditions, the agreement in the quantitative measurements (USAI and HRI) between different readers was excellent. A more interesting method would have required each examiner to acquire their own images on which to calculate the USAI and HRI. Secondly, the reference standard (histology of needle biopsy specimens) was prone to sampling errors because only a small portion of the liver was obtained. However, needle biopsy is probably the most practical reference standard currently available. Similarly, the USAI and HRI might not be representative of the entire liver but it does evaluate a much greater amount of liver tissue than biopsy. Thirdly, our study included he- patic donor candidates who were mostly young, healthy, and relatively lean adults. The number of patients with steatosis greater than $30 \%$ was relatively low and a large number of patients had little to no steatosis in our study population. If we had included more patients with variable degrees of steatosis from the general population, the results would have been more conclusive. However, obtaining liver biopsy for research purposes in such patients would not be feasible or ethical. Fourthly, the cut-off values for USAI and HRI for the diagnosis of steatosis $\geq 5 \%$ and $\geq 30 \%$, were not validated in a subgroup. Due to the small percentage of patients with steatosis, we had to put off the validation for a further study. Validation is possible in well-constructed future studies, overcoming the limitations of the retrospective design of our study.

\section{Conclusion}

Although the difference was statistically insignificant, HRI was superior to USAI for the diagnosis and quantitative estimation of hepatic steatosis, including accuracy and reproducibility. Therefore, HRI may be the preferred quantitative method for non-invasive US assessment of NAFLD.

\section{Conflict of interest: none}

\section{References}

1. Clark JM, Brancati FL, Diehl AM. Nonalcoholic fatty liver disease. Gastroenterology 2002;122:1649-1657.

2. Rosselli M, Lotersztajn S, Vizzutti F, Arena U, Pinzani M, Marra F. The metabolic syndrome and chronic liver disease. Curr Pharm Des 2014;20:5010-5024.

3. Hamaguchi M, Kojima T, Takeda N, et al. Nonalcoholic fatty liver disease is a novel predictor of cardiovascular disease. World J Gastroenterol 2007;13:1579-1584.

4. Bhala N, Angulo P, van der Poorten D, et al. The natural history of nonalcoholic fatty liver disease with advanced fibrosis or cirrhosis: an international collaborative study. Hepatology 2011;54:1208-1216.

5. Caldwell S, Argo C. The natural history of non-alcoholic fatty liver disease. Dig Dis 2010;28:162-168.

6. Ratziu V, Charlotte F, Heurtier A, et al. Sampling variability of liver biopsy in nonalcoholic fatty liver disease. Gastroenterology 2005;128:1898-1906.

7. Marshall RH, Eissa M, Bluth EI, Gulotta PM, Davis NK. Hepatorenal index as an accurate, simple, and effective tool in screening for steatosis. AJR Am J Roentgenol 2012;199:997-1002.

8. Palmentieri B, de Sio I, La Mura V, et al. The role of bright liver echo pattern on ultrasound B-mode examination in the diagnosis of liver steatosis. Dig Liver Dis 2006;38:485-489. 
9. Tobari M, Hashimoto E, Yatsuji S, Torii N, Shiratori K. Imaging of nonalcoholic steatohepatitis: advantages and pitfalls of ultrasonography and computed tomography. Intern Med 2009;48:739-746.

10. Strauss S, Gavish E, Gottlieb P, Katsnelson L. Interobserver and intraobserver variability in the sonographic assessment of fatty liver. AJR Am J Roentgenol 2007; 189: W320-W323.

11. Zwiebel WJ. Sonographic diagnosis of diffuse liver disease. Semin Ultrasound CT MR 1995;16:8-15.

12. Saadeh S, Younossi ZM, Remer EM, et al. The utility of radiological imaging in nonalcoholic fatty liver disease. Gastroenterology 2002;123:745-750.

13. Kwon HJ, Kim KW, Lee SJ, et al. Value of the ultrasound attenuation index for noninvasive quantitative estimation of hepatic steatosis. J Ultrasound Med 2013;32:229-235.

14. Webb M, Yeshua H, Zelber-Sagi S, et al. Diagnostic value of a computerized hepatorenal index for sonographic quantification of liver steatosis. AJR Am J Roentgenol 2009;192:909-914.

15. Martin-Rodriguez JL, Arrebola JP, Jimenez-Moleon JJ, Olea N, Gonzalez-Calvin JL. Sonographic quantification of a hepato-renal index for the assessment of hepatic steatosis in comparison with $3 \mathrm{~T}$ proton magnetic resonance spectroscopy. Eur J Gastroenterol Hepatol 2014;26:88-94.

16. Crowley H, Lewis WD, Gordon F, Jenkins R, Khettry U. Steatosis in donor and transplant liver biopsies. Hum Pathol 2000;31:1209-1213.
17. Contos MJ, Choudhury J, Mills AS, Sanyal AJ. The histologic spectrum of nonalcoholic fatty liver disease. Clin Liver Dis 2004;8:481-500.

18. Kleiner DE, Brunt EM, Van Natta M, et al. Design and validation of a histological scoring system for nonalcoholic fatty liver disease. Hepatology 2005;41:1313-1321.

19. Lee JY, Kim KM, Lee SG, et al. Prevalence and risk factors of non-alcoholic fatty liver disease in potential living liver donors in Korea: a review of 589 consecutive liver biopsies in a single center. J Hepatol 2007;47:239-244.

20. Perkins NJ, Schisterman EF. The inconsistency of "optimal" cutpoints obtained using two criteria based on the receiver operating characteristic curve. Am J Epidemiol 2006; 163:670-675.

21. Cleophas TJ, Droogendijk J, van Ouwerkerk BM. Validating diagnostic tests, correct and incorrect methods, new developments. Curr Clin Pharmacol 2008;3:70-76.

22. Bian H, Yan H, Zeng M, et al. Increased liver fat content and unfavorable glucose profiles in subjects without diabetes. Diabetes Technol Ther 2011;13:149-155.

23. Fleiss JL. Statistical methods for rates and proportions. New York: Wiley, 1981.

24. Xia MF, Yan HM, He WY, et al. Standardized ultrasound hepatic/renal ratio and hepatic attenuation rate to quantify liver fat content: an improvement method. Obesity (Silver Spring) 2012;20:444-452. 